\title{
Some properties of secantoptics of ovals
}

\author{
Witold Mozgawa • Magdalena Skrzypiec
}

Received: 14 February 2011 / Accepted: 30 May 2011 / Published online: 15 June 2011 (C) The Author(s) 2011. This article is published with open access at Springerlink.com

\begin{abstract}
In this paper we continue the investigation of further properties of secantoptics introduced and considered by Skrzypiec (Beiträge Algebra Geom. 49(1):205-215, 2008) and by Mozgawa and Skrzypiec (Bull. Belg. Math. Soc. Simon Stevin 16(3):435-445, 2009). We describe properties of secantoptics of ovals of constant width, prove a theorem on tangents to secantoptic and give the converse of the sine theorem for secantoptics.
\end{abstract}

Keywords Secantoptic $\cdot$ Isoptic $\cdot$ Secant $\cdot$ Support function

Mathematics Subject Classification (2000) $\quad 53 \mathrm{~A} 04 \cdot 53 \mathrm{C} 44$

\section{Introduction}

Let us first recall the notion of a secantoptic of an oval as it is defined in Skrzypiec (2008) and studied in Mozgawa and Skrzypiec (2009). Throughout this paper, by an oval we mean a plane, closed curve given by the equation

$$
z(t)=p(t) e^{i t}+\dot{p}(t) i e^{i t} \text { for } t \in[0,2 \pi)
$$

where $p(t)$, called the support function of an oval, is of class $C^{3}$ and the function $R(t)=p(t)+\ddot{p}(t)$ is positive for all $t \in[0,2 \pi)$. Note that the function $R(t)=$ $p(t)+\ddot{p}(t)$ is the curvature radius of the curve $z(t)$ at the point $t$, and that the support function $p(t)$ can be extended to a periodic function on $\mathbb{R}$ with the period $2 \pi$.

W. Mozgawa $(\varangle) \cdot$ M. Skrzypiec

Institute of Mathematics, UMCS, pl. M. Curie-Skłodowskiej 1, 20-031 Lublin, Poland

e-mail: mozgawa@hektor.umcs.lublin.pl

M. Skrzypiec

e-mail: mskrzypiec@hektor.umcs.lublin.pl 


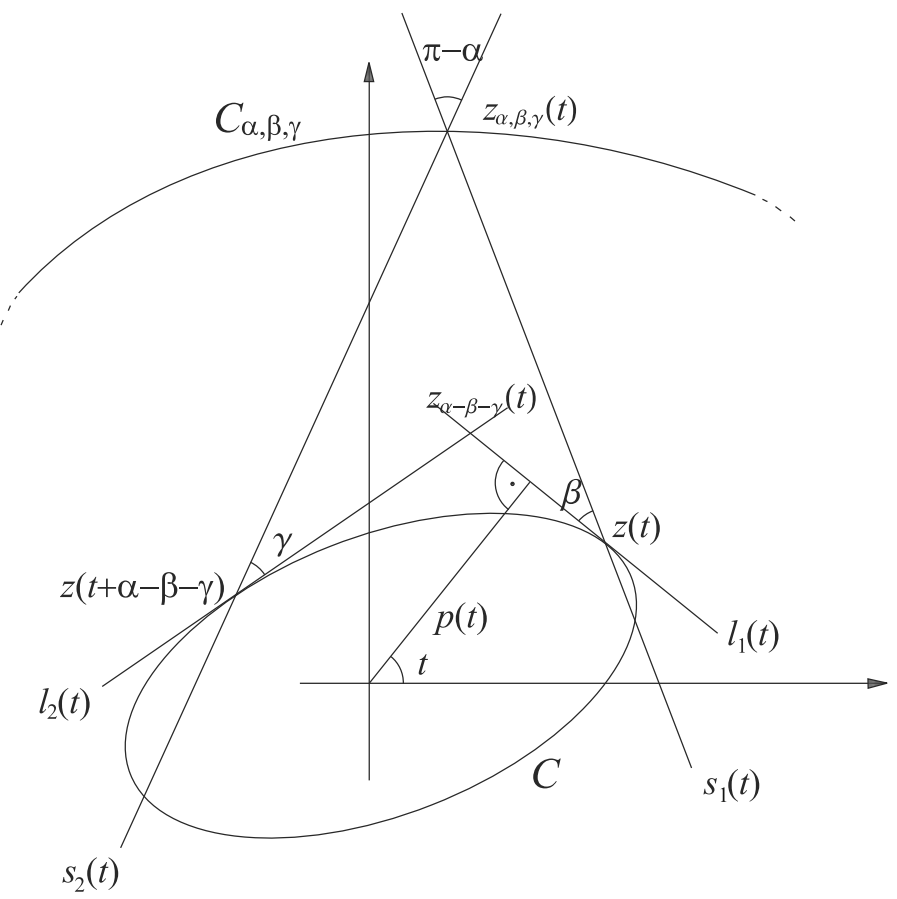

Fig. 1 Construction of a secantoptic $C_{\alpha, \beta, \gamma}$ of an oval $C$

Let $C$ be an oval and let $\beta \in[0, \pi), \gamma \in[0, \pi-\beta)$ and $\alpha \in(\beta+\gamma, \pi)$ be fixed angles. The set of intersection points $z_{\alpha, \beta, \gamma}(t)$ of $s_{1}(t)$ and $s_{2}(t)$ for every $t \in[0,2 \pi)$ forms a curve which we call a secantoptic $C_{\alpha, \beta, \gamma}$ of the oval $C$; see Fig. 1 . With the notations

$$
\begin{aligned}
q(t) & =z(t)-z(t+\alpha-\beta-\gamma), \\
b(t) & =\left[q(t), e^{i t}\right], \\
B(t) & =\left[q(t), i e^{i t}\right], \\
q(t) & =(B(t)-i b(t)) e^{i t}, \\
\lambda(t) & =\frac{b(t) \sin (\alpha-\beta)-B(t) \cos (\alpha-\beta)}{\sin \alpha}, \\
\mu(t) & =-\frac{b(t) \sin \beta+B(t) \cos \beta}{\sin \alpha},
\end{aligned}
$$

where $[v, w]=a d-b c$ for $v=a+b i$ and $w=c+d i$, we give the following parametrization of the secantoptic $C_{\alpha, \beta, \gamma}$ of the oval $C$ :

$$
z_{\alpha, \beta, \gamma}(t)=(p(t)+\lambda(t) \sin \beta+i(\dot{p}(t)+\lambda(t) \cos \beta)) e^{i t} \text { for } t \in[0,2 \pi)
$$

Secantoptics may be considered as isoptics of a pair of evolutoids of a given oval. Let us recall that an $\alpha$-isoptic $C_{\alpha}$ of a given curve $C$ is the locus of apices of a fixed angle 
$\pi-\alpha$, where $\alpha \in(0, \pi)$ formed by two supporting lines of $C$. More informations on isoptic curves can be found, for example, in Benko et al. (1990), Cieślak et al. (1991), Green (1950), Góźdź (1996), Góźdź et al. (1998), Matsuura (1993), Michalska (2003), Miernowski and Mozgawa (2001) and Szałkowski (2005, 2007). Isoptics of pair of curves were defined in Miernowski and Mozgawa (2001) and studied in Mozgawa and Skrzypiec (2009). Consider two evolutoids of an oval $C$

$$
\begin{aligned}
\Gamma_{-\beta}: \psi_{-\beta}(t) & =p(t+\beta) \cos \beta-\dot{p}(t+\beta) \sin \beta, \\
\Gamma_{\gamma}: \psi_{\gamma}(t) & =p(t-\gamma) \cos \gamma+\dot{p}(t-\gamma) \sin \gamma .
\end{aligned}
$$

The equation of an isoptic $C_{\alpha}^{\Gamma_{-\beta} \Gamma_{\gamma}}$, where $\beta \in[0, \pi), \gamma \in[0, \pi-\beta)$ and $\alpha \in$ $(\beta+\gamma, \pi)$, is given by

$$
z_{\alpha}^{\Gamma_{-\beta} \Gamma_{\gamma}}(t)=\psi_{-\beta}(t) e^{i t}+\left(\psi_{\gamma}(t+\alpha) \frac{1}{\sin \alpha}-\psi_{-\beta}(t) \cot \alpha\right) i e^{i t} .
$$

In Mozgawa and Skrzypiec (2009) we proved the following theorem.

Theorem 1.1 The isoptic $C_{\alpha}^{\Gamma_{-\beta} \Gamma_{\gamma}}$ and the secantoptic $C_{\alpha, \beta, \gamma}$ of a given oval C coincide if $\beta \in[0, \pi), \gamma \in[0, \pi-\beta)$, and $\alpha \in(\beta+\gamma, \pi)$.

It is very convenient to introduce the notations

$$
\begin{aligned}
L(t) & =R(t) \sin \beta+\lambda(t), \\
M(t) & =\mu(t)-R(t+\alpha-\beta-\gamma) \sin \gamma, \\
Q(t) & =M(t) i e^{i(t+\alpha-\beta)}-L(t) i e^{i(t-\beta)} .
\end{aligned}
$$

\section{Secantoptics of ovals of constant width}

The width in the direction $e^{i t}$ of an oval $C$ is defined as the distance between lines normal to this direction. If the width does not depend on $t$, the oval is called a curve of constant width. Curves of constant width and isoptics of such curves were studied in Cieślak et al. (1996), Miernowski and Mozgawa (1995), Robertson (1984) and Wegner (1989); see also the surveys (Chakerian and Groemer 1983; Heil and Martini 1993).

Theorem 2.1 If an oval $C$ is a curve of constant width $\Delta$, then the distance between points $z_{\alpha, \beta, \gamma}(t)$ and $z_{\alpha, \beta, \gamma}(t+\pi)$ on its secantoptic $C_{\alpha, \beta, \gamma}$ is constant and equal to $\frac{\Delta}{\sin \alpha} \sqrt{\cos ^{2} \beta+\cos ^{2} \gamma-2 \cos \alpha \cos \beta \cos \gamma}$.

Proof Let $C: z(t)=(p(t)+i \dot{p}(t)) e^{i t}$ be an oval of constant width $\Delta$. Then for any $t \in[0,2 \pi)$ we have

$$
\Delta=p(t)+p(t+\pi)
$$


If $t \mapsto z_{\alpha, \beta, \gamma}(t)$ is a parametrization of a secantoptic $C_{\alpha, \beta, \gamma}$ of an oval $C$, then

$$
\begin{aligned}
z_{\alpha, \beta, \gamma}(t)-z_{\alpha, \beta, \gamma}(t+\pi)= & {[(p(t)+p(t+\pi))+\sin \beta(\lambda(t)+\lambda(t+\pi))} \\
& +i((\dot{p}(t)+\dot{p}(t+\pi))+\cos \beta(\lambda(t)+\lambda(t+\pi)))] e^{i t} .
\end{aligned}
$$

To simplify this expression note that

$$
\begin{aligned}
\lambda(t)+\lambda(t+\pi)= & \frac{1}{\sin \alpha}[(p(t+\alpha-\beta-\gamma)+p(t+\alpha-\beta-\gamma+\pi)) \cos \gamma \\
& +(\dot{p}(t+\alpha-\beta-\gamma)+\dot{p}(t+\alpha-\beta-\gamma+\pi)) \sin \gamma \\
& -(p(t)+p(t+\pi)) \sin (\alpha-\beta)-(\dot{p}(t)+\dot{p}(t+\pi)) \cos (\alpha-\beta)],
\end{aligned}
$$

and from (2.1) we have

$$
p(t+\alpha-\beta-\gamma)+p(t+\alpha-\beta-\gamma+\pi)=\Delta
$$

and

$$
\dot{p}(t)+\dot{p}(t+\pi)=\frac{d \Delta}{d t}=0 .
$$

Hence we can replace the dependence on the support function of the oval $C$ in the expression $z_{\alpha, \beta, \gamma}(t)-z_{\alpha, \beta, \gamma}(t+\pi)$ by the dependence on the length of $C$ in the following way:

$$
\begin{aligned}
z_{\alpha, \beta, \gamma}(t)-z_{\alpha, \beta, \gamma}(t+\pi)= & \left(\Delta+\frac{\sin \beta}{\sin \alpha}(\Delta \cos \gamma-\Delta \cos (\alpha-\beta))\right. \\
& \left.+i \frac{\cos \beta}{\sin \alpha}(\Delta \cos \gamma-\Delta \cos (\alpha-\beta))\right) e^{i t}
\end{aligned}
$$

From this it follows that

$$
\left|z_{\alpha, \beta, \gamma}(t)-z_{\alpha, \beta, \gamma}(t+\pi)\right|=\frac{\Delta}{\sin \alpha} \sqrt{\cos ^{2} \beta+\cos ^{2} \gamma-2 \cos \alpha \cos \beta \cos \gamma} .
$$

Corollary 2.2 If $\beta=\gamma$, then

$$
\left|z_{\alpha, \beta, \beta}(t)-z_{\alpha, \beta, \beta}(t+\pi)\right|=\frac{\Delta \cos \beta}{\cos \frac{\alpha}{2}} .
$$

Theorem 2.3 Let $C: z(t)=(p(t)+i \dot{p}(t)) e^{i t}$ be an oval and let $\alpha-2 \beta$ be a number linearly independent on $\pi$ over $\mathbb{Q}$. If the distance between points $z_{\alpha, \beta, \beta}(t)$ and $z_{\alpha, \beta, \beta}(t+\pi)$ on its secantoptic $C_{\alpha, \beta, \beta}$ is constant, then the expression $\mid z(t)-$ $z(t+\pi) \mid$ is constant. 
Proof First we assume that $\beta \in[0, \pi)$ and $\gamma \in[0, \pi-\beta)$. Note that

$$
\begin{aligned}
& z_{\alpha, \beta, \gamma}(t)-z_{\alpha, \beta, \gamma}(t+\pi)=\left(\Delta(t)+\frac{\sin \beta}{\sin \alpha}(\Delta(t+\alpha-\beta-\gamma) \cos \gamma-\Delta(t) \cos (\alpha-\beta))\right. \\
& \left.+i \frac{\cos \beta}{\sin \alpha}(\Delta(t+\alpha-\beta-\gamma) \cos \gamma-\Delta(t) \cos (\alpha-\beta))\right) e^{i t}
\end{aligned}
$$

where $\Delta(t)=p(t)+p(t+\pi)$. Let

$$
D=\left|z_{\alpha, \beta, \gamma}(t)-z_{\alpha, \beta, \gamma}(t+\pi)\right|
$$

denote the constant length between points on the secantoptic $C_{\alpha, \beta, \gamma}$ of the oval $C$. Then there exists a function $t \mapsto \xi(t), 0<\xi(t)<\pi$, such that

$$
\begin{aligned}
\Delta(t)+ & \frac{\sin \beta}{\sin \alpha}(\Delta(t+\alpha-\beta-\gamma) \cos \gamma-\Delta(t) \cos (\alpha-\beta))=D \sin \xi(t), \\
& \frac{\cos \beta}{\sin \alpha}(\Delta(t+\alpha-\beta-\gamma) \cos \gamma-\Delta(t) \cos (\alpha-\beta))=D \cos \xi(t) .
\end{aligned}
$$

Using (2.6) and (2.5) we get

$$
\Delta(t+\alpha-\beta-\gamma)=\frac{\cos (\alpha-\beta)}{\cos \gamma}\left(D \cos \xi(t)\left(\frac{\sin \alpha}{\cos \beta \cos (\beta-\alpha)}-\tan \beta\right)+D \sin \xi(t)\right)
$$

If we insert (2.7) to the Eq. (2.6), we obtain

$$
\Delta(t)=D(\sin \xi(t)-\cos \xi(t) \tan \beta)
$$

and by using the substitution $t:=t+\alpha-\beta-\gamma$ we get

$$
\Delta(t+\alpha-\beta-\gamma)=\frac{D}{\cos \beta} \sin (\xi(t+\alpha-\beta-\gamma)-\beta) .
$$

From (2.7) we have

$$
\Delta(t+\alpha-\beta-\gamma)=\frac{D}{\cos \gamma} \sin (\xi(t)+\alpha-\beta)
$$

Using equations (2.9) and (2.10) we obtain the condition

$$
\frac{\sin (\xi(t+\alpha-\beta-\gamma)-\beta)}{\cos \beta}=\frac{\sin (\xi(t)+\alpha-\beta)}{\cos \gamma} .
$$

Suppose that the distance between points $z_{\alpha, \beta, \gamma}(t)$ and $z_{\alpha, \beta, \gamma}(t+\pi)$ on the secantoptic $C_{\alpha, \beta, \gamma}$ of an oval $C$ is constant. We would like to answer the question whether 
then $|z(t)-z(t+\pi)|$ is constant. For $\beta \neq \gamma$ it is still an open problem, but for $\beta=\gamma$ we get

$$
\frac{\sin (\xi(t+\alpha-2 \beta)-\beta)}{\cos \beta}=\frac{\sin (\xi(t)+\alpha-\beta)}{\cos \beta} .
$$

Since $\beta \in\left[0, \frac{\pi}{2}\right)$, then $\cos \beta \neq 0$. Therefore we obtain

$$
\sin (\xi(t+\alpha-2 \beta)-\beta)=\sin (\xi(t)+\alpha-\beta) .
$$

This condition is known from Cieślak et al. (1996). It is easy to see that

$$
\xi(t+\alpha-2 \beta)=\xi(t)+\alpha+2 \pi j
$$

or

$$
\xi(t+\alpha-2 \beta)=\pi-(\xi(t)+\alpha-2 \beta)+2 \pi k
$$

for certain $j, k \in \mathbb{Z}$. Since $0<\xi(t)<\pi$, then

$$
\xi(t+\alpha-2 \beta)=\xi(t)+\alpha
$$

or

$$
\xi(t+\alpha-2 \beta)+\xi(t)+\alpha-2 \beta=\pi .
$$

The function $\Delta(t)=p(t)+p(t+\pi)$ is periodic, and its period is equal to $2 \pi$. Hence

$$
\xi(t+2 \pi)=\xi(t)+2 \pi m,
$$

but since $0<\xi(t)<\pi$, then

$$
\xi(t+2 \pi)=\xi(t) .
$$

Conditions (2.13) and $0<\xi(t)<\pi$ are in contradiction, because from (2.13) we get

$$
\xi(t+n \delta)=\xi(t)+n \delta+2 \beta
$$

for $n \in \mathbb{N}$ and $\delta \in[0,2 \pi]$. If $n$ is sufficiently large, then the function $\xi(t)$ cannot be bounded. Therefore the conditions (2.14) and (2.16) must hold. Using the substitution $t:=t+\alpha-2 \beta$ in the formula (2.14) we obtain

$$
\xi(t+2(\alpha-2 \beta))+\xi(t+\alpha-2 \beta)+\alpha-2 \beta=\pi .
$$


If we subtract the formula (2.14) from (2.17), then

$$
\xi(t+2(\alpha-2 \beta))=\xi(t),
$$

what means that the function $\xi$ has two periods $2 \pi$ and $2(\alpha-2 \beta)$. Since we assumed in this theorem that the number $\alpha-2 \beta$ is linearly independent on $\pi$ over $\mathbb{Q}$, then $\xi$ has to be a constant.

\section{Theorem on tangents to secantoptic of an oval}

Let us fix a secantoptic $C_{\alpha, \beta, \gamma}$ of an oval $C$ and recall that we may consider it as an isoptic of pair of evolutoids of an oval $C$ whose support functions are

$$
\psi_{-\beta}(t)=p(t+\beta) \cos \beta-\dot{p}(t+\beta) \sin \beta
$$

and

$$
\psi_{\gamma}(t)=p(t-\gamma) \cos \gamma+\dot{p}(t-\gamma) \sin \gamma
$$

Then

$$
z_{\alpha, \beta, \gamma}(t)=z_{\alpha}^{\Gamma_{-\beta} \Gamma_{\gamma}}(t)=\psi_{-\beta}(t) e^{i t}+\left(\psi_{\gamma}(t+\alpha) \frac{1}{\sin \alpha}-\psi_{-\beta}(t) \cot \alpha\right) i e^{i t}
$$

and the first derivative of the parametrization of the secantoptic $C_{\alpha, \beta, \gamma}$ of the oval $C$ can be written as

$$
\dot{z}_{\alpha, \beta, \gamma}(t)=\dot{z}_{\alpha}^{\Gamma_{1} \Gamma_{2}}(t)=-L(t) e^{i t}+\rho_{1}(t) i e^{i t},
$$

where

$$
\begin{aligned}
L(t) & =-\dot{\psi}_{1}(t)-\psi_{1}(t) \cot \alpha+\psi_{2}(t+\alpha) \frac{1}{\sin \alpha}=b_{1}(t)-B_{1}(t) \cot \alpha, \\
M(t) & =-\psi_{1}(t) \frac{1}{\sin \alpha}-\dot{\psi}_{2}(t+\alpha)+\psi_{2}(t+\alpha) \cot \alpha=-\frac{B_{1}(t)}{\sin \alpha}, \\
\rho_{1}(t) & =\psi_{1}(t)+\dot{\psi}_{2}(t+\alpha) \frac{1}{\sin \alpha}-\dot{\psi}_{1}(t) \cot \alpha=B_{1}(t)+b_{1}(t) \cot \alpha
\end{aligned}
$$

and

$$
\begin{aligned}
& B_{1}(t)=\psi_{1}(t)-\psi_{2}(t+\alpha) \cos \alpha+\dot{\psi}_{2}(t+\alpha) \sin \alpha, \\
& b_{1}(t)=\psi_{2}(t+\alpha) \sin \alpha+\dot{\psi}_{2}(t+\alpha) \cos \alpha-\dot{\psi}_{1}(t) .
\end{aligned}
$$

Recall that

$$
Q(t)=M(t) i e^{i(t+\alpha-\beta)}-L(t) i e^{i(t-\beta)}=\left(B_{1}(t)-i b_{1}(t)\right) e^{i t} .
$$


Let us fix $\tau \in(0,2 \pi)$ and denote by $h^{\tau}(t)$ the function $h(t+\tau)$. Let $\angle(v, w)$ denote the angle between $v$ and $w$, while $\langle v, w\rangle$ is their scalar product.

Theorem 3.1 Let $C_{\alpha, \beta, \gamma}$ be a secantoptic of an oval $C$. Then we have the relation

$$
\angle\left(\dot{z}_{\alpha, \beta, \gamma}, \dot{z}_{\alpha, \beta, \gamma}^{\tau}\right)+\angle\left(Q, Q^{\tau}\right)=2 \tau .
$$

Proof Since

$$
\dot{z}_{\alpha, \beta, \gamma}^{\tau}(t)=e^{i t}\left(\left(-L^{\tau}(t) \cos \tau-\rho_{1}^{\tau}(t) \sin \tau\right)+i\left(\rho_{1}^{\tau}(t) \cos \tau-L^{\tau}(t) \sin \tau\right)\right),
$$

then

$$
\left\langle\dot{z}_{\alpha, \beta, \gamma}, \dot{z}_{\alpha, \beta, \gamma}^{\tau}\right\rangle=\cos \tau\left(L L^{\tau}+\rho_{1} \rho_{1}^{\tau}\right)+\sin \tau\left(L \rho_{1}^{\tau}-\rho_{1} L^{\tau}\right)
$$

We may use the functions $b_{1}(t)$ and $B_{1}(t)$ to rewrite the above expression to the form

$$
\left\langle\dot{z}_{\alpha, \beta, \gamma}, \dot{z}_{\alpha, \beta, \gamma}^{\tau}\right\rangle=\frac{b_{1} b_{1}^{\tau}+B_{1} B_{1}^{\tau}}{\sin ^{2} \alpha} \cos \tau+\frac{b_{1} B_{1}^{\tau}-B_{1} b_{1}^{\tau}}{\sin ^{2} \alpha} \sin \tau
$$

and then use (3.1). The further calculations in this proof are the same as in Cieślak et al. (1996). We will recall them there. A vector $Q^{\tau}(t)$ can be written as

$$
Q^{\tau}(t)=e^{i t}\left(B_{1}^{\tau}(t) \cos \tau+b_{1}^{\tau}(t) \sin \tau+i\left(B_{1}^{\tau}(t) \sin \tau-b_{1}^{\tau}(t) \cos \tau\right)\right),
$$

hence we can compute

$$
\left\langle Q, Q^{\tau}\right\rangle=\left(B_{1} B_{1}^{\tau}+b_{1} b_{1}^{\tau}\right) \cos \tau-\left(b_{1} B_{1}^{\tau}-B_{1} b_{1}^{\tau}\right) \sin \tau
$$

and

$$
\left[Q, Q^{\tau}\right]=\left(b_{1} B_{1}^{\tau}-B_{1} b_{1}^{\tau}\right) \cos \tau+\left(B_{1} B_{1}^{\tau}+b_{1} b_{1}^{\tau}\right) \sin \tau \text {. }
$$

Notice that

$\left[Q, Q^{\tau}\right] \sin 2 \tau+\left\langle Q, Q^{\tau}\right\rangle \cos 2 \tau=\left(B_{1} B_{1}^{\tau}+b_{1} b_{1}^{\tau}\right) \cos \tau+\left(b_{1} B_{1}^{\tau}-B_{1} b_{1}^{\tau}\right) \sin \tau$,

and we get the dependence

$$
\left\langle\dot{z}_{\alpha, \beta, \gamma}, \dot{z}_{\alpha, \beta, \gamma}^{\tau}\right\rangle \sin ^{2} \alpha=\left[Q, Q^{\tau}\right] \sin 2 \tau+\left\langle Q, Q^{\tau}\right\rangle \cos 2 \tau
$$

Using

$$
\begin{aligned}
& \langle v, w\rangle=|v||w| \cos \angle(v, w), \\
& {[v, w]=|v||w| \sin \angle(v, w)}
\end{aligned}
$$


and $\left|\dot{z}_{\alpha, \beta, \gamma}\right| \sin \alpha=|Q|$, we have

$$
\cos \angle\left(\dot{z}_{\alpha, \beta, \gamma}, \dot{z}_{\alpha, \beta, \gamma}^{\tau}\right)=\cos \left(2 \tau-L\left(Q, Q^{\tau}\right)\right)
$$

Therefore either

$$
L\left(\dot{z}_{\alpha, \beta, \gamma}, \dot{z}_{\alpha, \beta, \gamma}^{\tau}\right)+L\left(Q, Q^{\tau}\right)=2 \tau
$$

or

$$
L\left(\dot{z}_{\alpha, \beta, \gamma}, \dot{z}_{\alpha, \beta, \gamma}^{\tau}\right)+2 \tau=L\left(Q, Q^{\tau}\right)
$$

or

$$
\angle\left(\dot{z}_{\alpha, \beta, \gamma}, \dot{z}_{\alpha, \beta, \gamma}^{\tau}\right)=2 \pi-2 \tau+L\left(Q, Q^{\tau}\right)
$$

If $\tau \rightarrow 0$, then $\angle\left(\dot{z}_{\alpha, \beta, \gamma}, \dot{z}_{\alpha, \beta, \gamma}^{\tau}\right) \rightarrow 0$ and $\angle\left(Q, Q^{\tau}\right) \rightarrow 0$. On the other hand, if $\tau \rightarrow 2 \pi$, then $L\left(\dot{z}_{\alpha, \beta, \gamma}, \dot{z}_{\alpha, \beta, \gamma}^{\tau}\right) \rightarrow 2 \pi$ and $L\left(Q, Q^{\tau}\right) \rightarrow 2 \pi$. Hence we obtain (3.3).

If $\tau=\pi$, then, as it was shown for isoptics in Cieślak et al. (1996), we get the following corollaries.

Corollary 3.2 Let $C_{\alpha, \beta, \gamma}$ be a secantoptic of an oval $C$. Then

$$
L\left(\dot{z}_{\alpha, \beta, \gamma}(t), \dot{z}_{\alpha, \beta, \gamma}(t+\pi)\right)+L(Q(t), Q(t+\pi))=2 \pi .
$$

Corollary 3.3 Let $C_{\alpha, \beta, \gamma}$ be a secantoptic of an oval $C$. Then the vector $\dot{z}_{\alpha, \beta, \gamma}$ is parallel to the vector $\dot{z}_{\alpha, \beta, \gamma}^{\tau}$ if and only if the vector $Q$ is parallel to $Q^{\tau}$.

\section{The converse to the sine theorem for secantoptics}

In Skrzypiec (2008) we have proved the sine theorem for secantoptics. Now we want to give the converse theorem.

Theorem 4.1 Let $C: z(t)=p(t) e^{i t}+\dot{p}(t) i e^{i t}$ be a given oval such that $p$ is of class $C^{3}$ and let $\Gamma$ be a regular Jordan curve. Suppose that there exists a differentiable function $\varphi: \mathbb{R} \mapsto \mathbb{R}$ such that

(1) through a point $z(t)$ of an oval $C$ we draw a tangent line and rotate it around a tangency point about the angle $-\beta$, where $\beta \in[0, \pi)$ (the obtained secant we denote by $\left.s_{1}(t)\right)$,

(2) through a point $z(\varphi(t))$ of a curve $C$ we draw a tangent line and rotate it around a tangency point about the angle $\gamma$, where $\gamma \in[0, \pi-\beta$ ) (the obtained secant we denote by $\left.s_{2}(t)\right)$,

(3) secants $s_{1}(t)$ and $s_{2}(t)$ intersect by forming an angle $\alpha(t) \in(\beta+\gamma, \pi)$ for each $t \in[0,2 \pi)$ in a certain point on the curve $\Gamma$, 


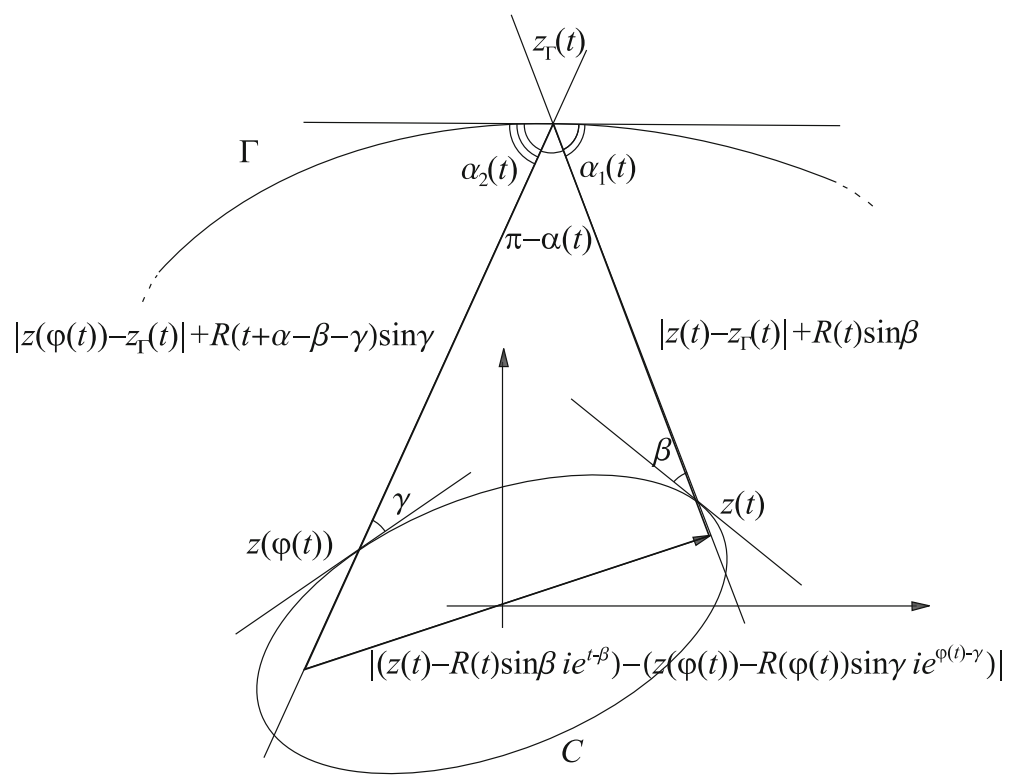

Fig. 2 Notations used for Theorem 4.1

(4) for each $t \in[0,2 \pi)$ the sine formula

$$
\begin{aligned}
& \frac{\left|z(t)-z_{\Gamma}(t)\right|+R(t) \sin \beta}{\sin \alpha_{1}(t)}=\frac{\left|z(\varphi(t))-z_{\Gamma}(t)\right|+R(\varphi(t)) \sin \gamma}{\sin \alpha_{2}(t)} \\
& =\frac{\left|\left(z(t)-R(t) \sin \beta i e^{t-\beta}\right)-\left(z(\varphi(t))-R(\varphi(t)) \sin \gamma i e^{\varphi(t)+\gamma}\right)\right|}{\sin \alpha(t)}
\end{aligned}
$$

holds, where $\alpha_{1}(t)$ and $\alpha_{2}(t)$ are the angles which the tangent line to the curve $\Gamma$ form with the secants $s_{1}(t)$ and $s_{2}(t)$, respectively (Fig. 2$)$.

Then $\Gamma$ is a secantoptic $C_{\alpha, \beta, \gamma}$ of an oval $C$.

Proof Note that the function $\varphi(t)$ defines the function $\alpha(t)$ which satisfy a condition $0<\alpha(t)<\pi$ and determine the angle between considered secants by the formula

$$
\varphi(t)=t+\alpha(t)-\beta-\gamma
$$

Therefore we can express the equation of the curve $\Gamma$ by using the support function of the given oval $C$. Namely,

$$
z_{\Gamma}(t)=\left(p(t)+\lambda_{\Gamma}(t) \sin \beta+i\left(\dot{p}(t)+\lambda_{\Gamma}(t) \cos \beta\right)\right) e^{i t},
$$


where

$$
\begin{aligned}
\lambda_{\Gamma}(t)= & \frac{1}{\sin \alpha(t)}(p(t+\alpha(t)-\beta-\gamma) \cos \gamma+\dot{p}(t+\alpha(t)-\beta-\gamma) \sin \gamma \\
& -\dot{p}(t) \sin (\alpha(t)-\beta)-p(t) \cos (\alpha(t)-\beta)) .
\end{aligned}
$$

Note that the value of the function $\lambda_{\Gamma}$ for each $t \in[0,2 \pi)$ is a length of a line segment connecting the points $z(t)$ and $z_{\Gamma}(t)$. Similarly, the value of the function

$$
\begin{aligned}
\mu_{\Gamma}(t)= & \frac{-1}{\sin \alpha(t)}(-p(t+\alpha(t)-\beta-\gamma) \cos (\alpha(t)-\gamma) \\
& +\dot{p}(t+\alpha(t)-\beta-\gamma) \sin (\alpha(t)-\gamma)-\dot{p}(t) \sin \beta+p(t) \cos \beta)
\end{aligned}
$$

describes the length of a line segment $\left|z(t+\alpha(t)-\beta-\gamma) z_{\Gamma}(t)\right|$. Since we assume that the curve $\Gamma$ satisfies the sine formula (4.1), then

$$
\begin{aligned}
& \sin \alpha_{1}(t)=-\frac{\left[\dot{z}_{\Gamma}(t), i e^{i(t-\beta)}\right]}{\left|\dot{z}_{\Gamma}(t)\right|}, \\
& \sin \alpha_{2}(t)=\frac{\left[\dot{z}_{\Gamma}(t), i e^{i(t+\alpha(t)-\beta)}\right]}{\left|\dot{z}_{\Gamma}(t)\right|},
\end{aligned}
$$

where

$$
\dot{z}_{\Gamma}(t)=\left(\dot{\lambda}_{\Gamma}(t) \sin \beta-\lambda_{\Gamma}(t) \cos \beta+i\left(R(t)+\lambda_{\Gamma}(t) \sin \beta+\dot{\lambda}_{\Gamma}(t) \cos \beta\right)\right) e^{i t} .
$$

Hence

$$
\begin{gathered}
{\left[\dot{z}_{\Gamma}(t), i e^{i(t-\beta)}\right]=-\left(\lambda_{\Gamma}(t)+R(t) \sin \beta\right),} \\
{\left[\dot{z}_{\Gamma}(t), i e^{i(t+\alpha(t)-\beta)}\right]=\left(R(t+\alpha(t)-\beta-\gamma) \sin \gamma-\mu_{\Gamma}(t)\right)(1+\dot{\alpha}(t)) .}
\end{gathered}
$$

With the notations

$$
\begin{aligned}
L_{\Gamma}(t) & =\lambda_{\Gamma}(t)+R(t) \sin \beta, \\
M_{\Gamma}(t) & =\mu_{\Gamma}(t)-R(t+\alpha(t)-\beta-\gamma) \sin \gamma
\end{aligned}
$$

we can rewrite the sine formula in the form

$$
\frac{L_{\Gamma}(t)}{\left[i e^{i(t-\beta)}, \dot{z}_{\Gamma}(t)\right]}=\frac{-M_{\Gamma}(t)}{\left[\dot{z}_{\Gamma}(t), i e^{i(t+\alpha(t)-\beta)}\right]} .
$$

Hence we have the formula

$$
\frac{L_{\Gamma}(t)}{L_{\Gamma}(t)}=\frac{-M_{\Gamma}(t)}{-M_{\Gamma}(t)(1+\dot{\alpha}(t))},
$$


which can be simplified to

$$
\begin{aligned}
-L_{\Gamma}(t) M_{\Gamma}(t)(1+\dot{\alpha}(t)) & =-L_{\Gamma}(t) M_{\Gamma}(t), \\
L_{\Gamma}(t) M_{\Gamma}(t) \dot{\alpha}(t) & =0 .
\end{aligned}
$$

The functions $L_{\Gamma}(t)$ and $M_{\Gamma}(t)$ have positive values for all $t$, hence $\alpha(t)=$ const.

Open Access This article is distributed under the terms of the Creative Commons Attribution Noncommercial License which permits any noncommercial use, distribution, and reproduction in any medium, provided the original author(s) and source are credited.

\section{References}

Benko, K., Cieślak, W., Góźdź, S., Mozgawa, W.: On isoptic curves. An. Ştiinţ. Univ. Al. I. Cuza Iaşi Secţ. I a Mat. 36(1), 47-54 (1990)

Chakerian, G.D., Groemer, H.: Convex bodies of constant width. In: Gruber, P.M., Wills, J.M. (eds.) Convexity and its Applications, pp. 49-96. Birkhäuser, Basel (1983)

Cieślak, W., Miernowski, A., Mozgawa, W.: Isoptics of a closed strictly convex curve. In: Global Differential Geometry and Global Analysis (Berlin, 1990), Lecture Notes in Mathematics, vol. 1481, pp. 28-35. Springer, Berlin (1991)

Cieślak, W., Miernowski, A., Mozgawa, W.: Isoptics of a closed strictly convex curve II. Rend. Sem. Mat. Univ. Padova 96, 37-49 (1996)

Green, J.W.: Sets subtending a constant angle on a circle. Duke Math. J. 17, 263-267 (1950)

Góźdź, S.: On Jordan plane curves which are isoptics of an oval. An. Ştiinţ. Univ. Al. I. Cuza Iaşi Secţ. I a Mat. 42(1), 127-130 (1996)

Góźdź, S., Miernowski, A., Mozgawa, W.: Sine theorem for rosettes. An. Ştiinţ. Univ. Al. I. Cuza Iaşi Secţ. I a Mat. 44(2), 385-394 (1998)

Heil, E., Martini, H.: Special convex bodies. In: Gruber, P.M., Wills, J.M. (eds.) Handbook of Convexity, vol. A, pp. 347-385. North-Holland, Amsterdam (1993)

Matsuura, S.: On non-convex curves of constant angle. Lect. Notes Math. 1540, 251-268 (1993)

Michalska, M.: A sufficient condition for the convexity of the area of an isoptic curve of an oval. Rend. Semin. Mat. Univ. Padova 110, 161-169 (2003)

Miernowski, A., Mozgawa, W.: Isoptics of rosettes and rosettes of constant width. Note Mat. 15(2), 203213 (1995)

Miernowski, A., Mozgawa, W.: Isoptics of pairs of nested closed strictly convex curves and Crofton-type formulas. Beiträge Algebra Geom. 42(1), 281-288 (2001)

Mozgawa, W., Skrzypiec, M.: Crofton formulas and convexity condition for secantoptics. Bull. Belg. Math. Soc. Simon Stevin 16(3), 435-445 (2009)

Robertson, S.A.: Smooth curves of constant width and transnormality. Bull. Lond. Math. Soc. 16, 264274 (1984)

Skrzypiec, M.: A note on secantoptics. Beiträge Algebra Geom. 49(1), 205-215 (2008)

Szałkowski, D.: Isoptics of open rosettes. Ann. Univ. Mariae Curie-Skłodowska, Sect. A 59, 119-128 (2005)

Szałkowski, D.: Isoptics of open rosettes. II. An Ştiinţ. Univ. Al. I. Cuza Iaşi Secţ. I a Mat. 53(1), 167176 (2007)

Wegner, B.: Some global properties and constructions for closed curves in the plane. Geom. Dedicata 29(3), 317-326 (1989) 